\title{
Galaxies in Selected Fields of the Second Byurakan Survey
}

\author{
S. A. Hakopian, S. K. Balayan \\ Byurakan Astrophysical Observatory, Byurakan 378433, Armenia
}

\begin{abstract}
.
The current state of investigation of galaxies in seven fields of the Second Byurakan Sky Survey $(S B S)$ is presented. These fields have been selected by the results of completeness estimation of the samples of galaxies in 65 fields.

Observations of the $S B S$ faint candidate galaxies are carried out to complete spectroscopy of galaxies in the selected fields. Currently in one $S B S$ field, with coordinates of center $\alpha=15^{h} 30^{m}$ and $\delta=+59^{\circ}$, the spectra of all galaxies have been obtained and reduced. Besides the redshift and spectral classification, these data allow estimates of the quality of object selection in the Second Byurakan Survey at faint magnitudes.
\end{abstract}

\section{Introduction}

Due to an improved observational technique (Stepanian 1994a), the Second Byurakan Sky Survey (Markarian et al 1983, Markarian \& Stepanian 1983) extends a small part (1000 $\mathrm{deg}^{2}$ ) of the First Byurakan Survey (Markarian 1967) to deeper magnitudes, up to $19^{m}-19.5^{m}$. In particular, differently from the First Byurakan Survey, during the Second Survey three objective prisms, namely 1.5, 3 , and 4 degrees have been used. In total, photographic plates for 65 fields, each $16 \mathrm{deg}^{2}$, have been obtained with the $1.5^{\circ}$ prism. Only ten of these fields have been observed with the two other prisms also (Stepanian 1994b).

The relative uniformity of samples of objects selected by eye inspection of photographic plates of the survey fluctuates from field to field. It depends mainly on the number of plates obtained in the field and the limiting magnitudes. For the subsequent more detailed researches of samples of galaxies, we selected seven fields, for which:

- photographic plates have been obtained with the three objective prisms;

- the limiting value $19^{m}-19.5^{m}$ has been reached;

- large amount of follow-up slit spectroscopy has been carried out.

\section{The samples of galaxies in selected $S B S$ fields}

Among 3000 objects selected and catalogued during the $S B S$ (Stepanian 1994a) more than 1200 are galaxies with $U V$-excess, emission features and other peculiarities. 


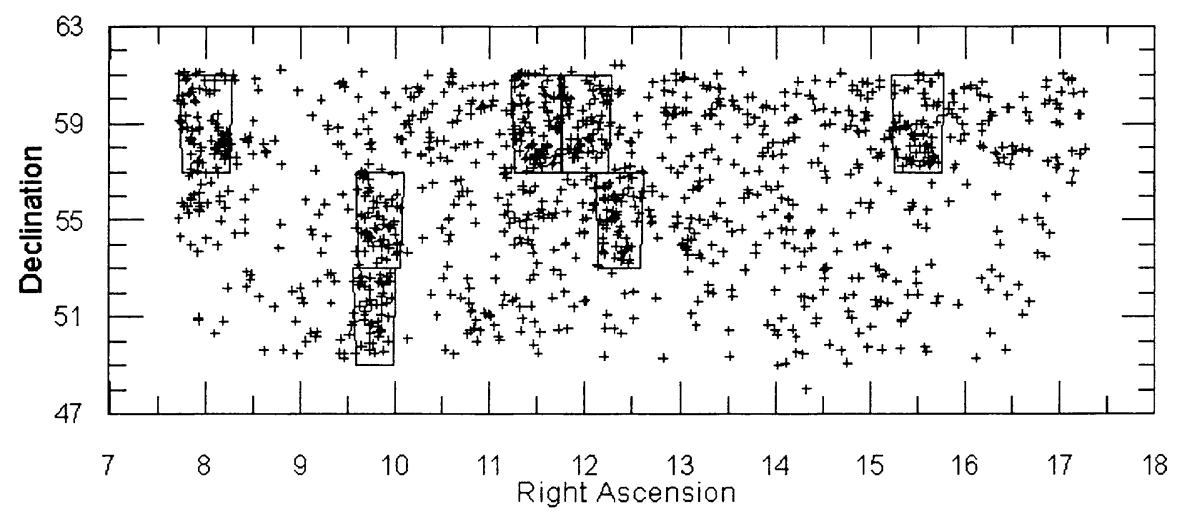

Figure 1. Distribution of $S B S$ galaxies in the sky and location of seven selected fields.

Fig.1 illustrates the distribution of all $S B S$ galaxies in the sky and location of the seven fields. More precise coordinates of the field centers are given in the second column of Table 1 . The next two columns show accordingly the number of galaxies selected in each sample and those with known redshift.

Table 1. Main data on the selected fields

\begin{tabular}{lccccc}
\hline $\mathrm{N}$ & $\begin{array}{c}\text { R.A. } \\
(1950)\end{array}$ & $\begin{array}{c}\text { Number of } \\
\text { Galaxies }\end{array}$ & $\begin{array}{c}\text { With known } \\
\text { redshift }\end{array}$ & $\begin{array}{c}m_{\text {comp }} \\
\text { (of } V / V_{\max } \text { test) }\end{array}$ \\
\hline 1 & $08^{h} 00^{m}+59^{\circ}$ & 88 & 74 & 16.0 \\
2 & $09^{h} 47^{m}+51^{\circ}$ & 54 & 38 & 18.0 \\
3 & $09^{h} 50^{m}+55^{\circ}$ & 67 & 48 & 16.5 \\
4 & $11^{h} 30^{m}+59^{\circ}$ & 96 & 81 & 15.5 \\
5 & $12^{h} 00^{m}+59^{\circ}$ & 72 & 20 & 18.0 \\
6 & $12^{h} 22^{m}+55^{\circ}$ & 74 & 49 & 17.5 \\
7 & $15^{h} 30^{m}+59^{\circ}$ & 68 & 68 & 18.0 \\
\hline Total & $112 \mathrm{deg}^{2}$ & 519 & 378 & 16.0 \\
\hline
\end{tabular}

For the given numbers of galaxies we applied the classical $V / V_{\max }$ test. In the last column of the Table 1 the values of completeness level are given for each sample of galaxies and their total. Fig. 4c shows the distribution of available apparent magnitudes, used during the calculations, defined in most cases with an accuracy of $0.5^{m}$.

The areas of the seven fields together compose only a tenth of the $S B S$. At the same time more than forty percent of the whole sample of $S B S$ galaxies, approximately 500 , have been selected in this part. We note that only one small cluster of galaxies, Abell634, is in the indicated bounds but not reflected in our sample. All of the above about the good quality of photographic plates and 

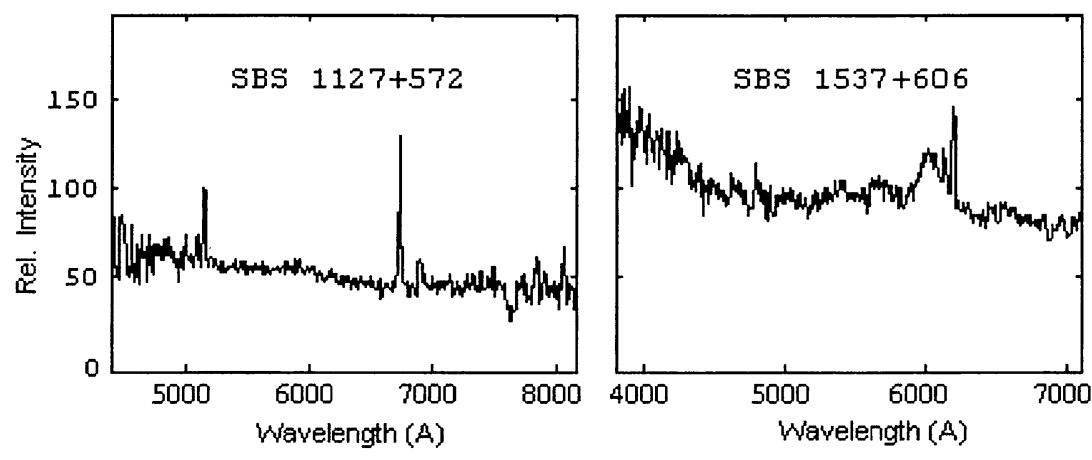

Figure 2. a) The first CCD spectra obtained at the Byurakan 2.6-m telescope. b) Spectra of the Sy1 galaxy obtained at the Russian 6-m telescope.

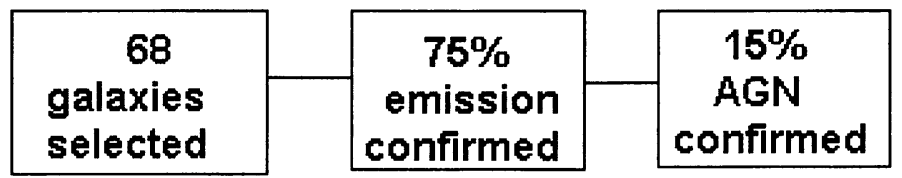

Figure 3. Schematic illustration of the sample content.

comparatively high percent of selected galaxies in the seven fields shows that their complete slit spectroscopy will help to clarify potentialities of the Survey.

\section{Spectral observations in the seventh field}

We carried out spectral observations from 1996 trying to obtain missing slit spectra for galaxies in the selected fields at the 6-m telescope of the Russian Special Astrophysical Observatory, and from March 1998 with the 2.6-m telescope of the Byurakan Observatory. One of the first CCD spectra obtained with the 2.6-m telescope with a grism is illustrated in Fig. 2a.

Up to now we have obtained 90 spectra of the 70 faintest galaxies in the samples with the longslit spectrograph at the 6-m telescope (Hakopian \& Balayan 1997, Balayan \& Hakopian 1999). By adding 35 of them to the previous spectroscopy (Stepanian et al 1993a, 1993b), the field numbered as seventh became the first field of the SBS with complete slit spectroscopy of its galaxies. The analysis of data obtained for this field sample is especially interesting, because it is one of the three with the highest level of completeness (see Table 1).

The follow-up spectroscopy confirms the emission nature of approximately $75 \%$ of this subsample of galaxies, with $15 \%$ of AGN galaxies among them. Fig. 3 schematically illustrates these results. 
One of the objects, $S B S 1537+606$ (Fig. $2 \mathrm{~b}$ ), turned out to be one of the most distant Sy1 type galaxies in the Survey, with redshift $z=0.2378$. In all there are seven AGNs in the seventh field - four of Sy1 and three of Sy2 type.

The most probable values of the Survey objects' redshifts, to be confirmed by follow-up observations, can be estimated, knowing the limits of spectral ranges obtained with combinations of prisms and photographic emulsions used (Stepanian 1994b), and the main criteria of object selection. In particular, all objects with redshifts up to 0.05 , that is $200 \mathrm{Mpc}$ at a Hubble constant of 75 $\mathrm{km} \mathrm{s}^{-1} \mathrm{Mpc}^{-1}$, could be selected by eye inspection, if even one of the emissionlines of hydrogen $\mathrm{H}_{\alpha}, \mathrm{H}_{\beta}$ and/or oxygen $5007 \AA\left(\mathrm{N}_{1}\right)$, is noticeable on the lowdispersion spectra.

New slit spectra of the faintest galaxies have revealed a number of absorptionline galaxies. According to Markarian et al (1986), all these objects were selected with the erroneous assumption to have one of the above mentioned emission lines. Figure 4a shows the redshift distribution of all galaxies in the seventh field, including those with absorption lines (shaded).

\section{Current results}

Figure $4 \mathrm{~b}$ shows the distribution of all known redshifts of galaxies in selected fields, including the seventh. Their apparent magnitudes are given by the unshaded part of the histogram, presented by Figure 4c. Figure $4 \mathrm{~d}$ shows the distribution of absolute magnitudes, calculated with value of the Hubble constant $75 \mathrm{~km} \mathrm{~s}^{-1} \mathrm{Mpc}^{-1}$. A comparison of distributions given can be done also with those for the whole sample of $S B S$ galaxies, using published data (e.g. Carrasco et al, 1997).

We found counterparts in different spectral ranges for about 120 galaxies in selected fields, using mainly the CATS database (Verkhodanov et al 1997). Here we shall dwell only on identifications of galaxies of AGN nature, namely 26 galaxies of Sy type.

There are $14 \mathrm{Sy} 1$ and $12 \mathrm{Sy} 2$ type galaxies in seven fields. All of the Sy2 objects radiate in radio and some of them in IR ranges. Eight Sy1 objects have counterparts in the $X$-ray range and some of them in other spectral ranges, too. It is interesting that the remaining 6 Syl galaxies, which have no counterparts in any other spectral ranges, are the most distant in this subsample. It seems that there is some threshold at redshifts about 0.12 , after which our Sy1 galaxies are not discovered in other parts of the spectrum. Taking into $\mathrm{w}$ account that such a distance effect is apparent only for a small sample, and could be affected by the lack of spatial resolution of $X$-ray telescopes, we have to learn more about their morphology and space orientation for more comprehensive conclusions.

\section{Summary}

We have completed slit spectroscopy of galaxies in one of the seven fields of the Second Byurakan Survey, selected for further investigations. The faint tail has been observed for the first time, enabling the evaluation of quality of object selection at limiting magnitudes of the Survey. The relatively high level of completeness confirms the comparison of data obtained for three subsamples of 


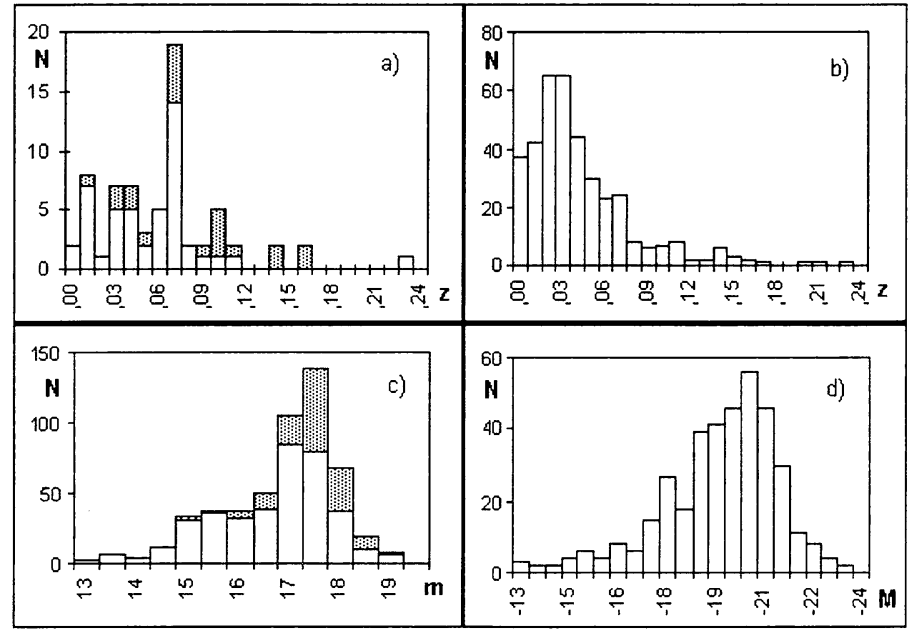

Figure 4. a) Redshift distribution for 68 galaxies in the seventh field; absorption-line galaxies are shaded, b) Histogram of known redshifts of 378 galaxies in selected fields. c) The distribution of apparent magnitudes of all 519 galaxies selected in seven fields; those with known $\mathrm{z}$ are unshaded. d) The distribution of absolute magnitudes of 378 galaxies in selected fields. 
$S B S$ galaxies - in the seventh field, in the seven fields, and in the whole $S B S$ area. The value of the redshift distribution peak of the seventh field sample, shifted as compared with two others, and the unusually high number of Sy type galaxies (0.4persq.deg.) can be considered as an upper limit of the potentialities of the $S B S$.

Acknowledgments. The authors are very thankful for useful advice to Dr. J. A. Stepanian, and for discussions with Dr. S. N. Dodonov and Prof. E. Ye. Khachikian.

\section{References}

Balayan S. K., \& Hakopian S. A. 1999, Astrofizika, in press

Carrasco, L., Serrano, A., Tovmassian, H.M., Stepanian, J.A., Chavushian, V.H., \& Erastova, L. K. 1997, AJ, 113, 1527

Hakopian S. A., \& Balayan S. K. 1997, Astrofizika, 40, 169

Markarian, B.E. 1967, Astrofizika, 3, 55

Markarian, B.E., Lipovetsky, V.A., \& Stepanian, J. A. 1983, Astrofizika, 18,29

Markarian, B.E., \& Stepanian, J. A. 1983, Astrofizika, 19, 639

Markarian B. E., Stepanian, J. A, \& Erastova L. K. 1986, Astrofizika, 25, 345

Stepanian, J. A., Lipovetsky, V. A., Erastova, L. K., Shapovalova, A. I., \& Hakopian, S. A. 1993a, Bull. Spec. Astrophys. Obs., (Izv. SAO), 35, 24

Stepanian, J.A., Lipovetsky, V.A., Erastova, L. K., \& Hakopian, S. A., Izotov, Yu. I., \& Guseva, N. G. 1993b, Bull. Spec. Astrophys. Obs., (Izv. SAO), 35,38

Stepanian, J. A. 1994a, Doctoral dissertation, Nizhnij Arkhys

Stepanian, J.A. 1994b, H.T.MacGillivray et al.(eds), Astronomy from WideField Imaging, p.731

Verkhodanov, O. V., Trushkin S. A., Andernach H., \& Chernenkov V.N. 1997, The CATS database to operate with astrophysical catalogs.In Proc. of the "Astronomical Data Analysis Software and Systems VI", ed. G.Hunt \& H. E.Payne, ASP Conference Series. V. 125, P. 32 